\title{
Acidificação do solo sob fertilização nitrogenada de longo prazo em campo nativo com introdução de azevém
}

\author{
Soil acidification under long-term nitrogen fertilization in a native pasture with ryegrass introduction
Diego Cecagno*, Sérgio Ely Valadão Gigante de Andrade Costa, Ibanor Anghinoni, Daniel Martins Brambilla e Carlos Nabinger

Universidade Federal do Rio Grande do Sul, Porto Alegre, RS, Brasil. *Autor para correspondência: dcecagno@hotmail.com.

Submissão: 27/08/2017 / Aceite: 25/02/2019

\begin{abstract}
RESUMO
A pastagem nativa é uma importante fonte de alimentos na produção de bovinos e ovinos no bioma Pampa. A introdução do azevém e a fertilização nitrogenada são alternativas que visam intensificar a exploração pecuária de forma mais sustentável. Este trabalho teve por objetivo avaliar os efeitos de doses de nitrogênio em cobertura em pastagem nativa com introdução de azevém nos indicadores de acidez do solo. O experimento consistiu de uma sucessão secundária da pastagem natural submetida ao pastejo contínuo. A área foi calcareada, e foram utilizadas três doses de nitrogênio em cobertura, correspondendo a 0, 100 e $200 \mathrm{~kg} \mathrm{ha}^{-1}$ ano $^{-1}$ de nitrogênio. Foram realizadas avaliação de saturação por bases, saturação por alumínio e pH nas camadas de 0-10 e 10-20 cm. Na camada de 0-10 cm, para cada $100 \mathrm{~kg} \mathrm{ha}^{-1} \mathrm{ano}^{-1}$ de nitrogênio aplicado em cobertura, o pH decresceu 0,27 unidades. Na camada de $10-20 \mathrm{~cm} \circ \mathrm{pH}$ decresceu de 5,3 até 4,7 e 4,6 com a aplicação de nitrogênio na dose de 100 e $200 \mathrm{~kg} \mathrm{ha}^{-1} \mathrm{ano}^{-1}$, respectivamente. A fertilização nitrogenada de longo prazo em campo nativo com introdução de azevém resultou em acidificação do solo até a profundidade de $20 \mathrm{~cm}$.
\end{abstract}

PALAVRAS-CHAVE: indicadores de acidez, pastagem natural, calagem.

\begin{abstract}
Native pastures are very important food sources for bovine and sheep production in the Pampa biome. Ryegrass introduction and nitrogen fertilization are alternatives aimed at a more sustainable intensification of livestock production. The objective of this study was to evaluate the effects of doses of nitrogen fertilizer in native pasture with ryegrass introduction on soil chemical acidity indicators. The experiment consists of a secondary native pasture under continuous grazing. The area was limed, and three different nitrogen rates $\left(0,100\right.$, and $200 \mathrm{~kg} \mathrm{ha}^{-1}$ year ${ }^{-1}$ of nitrogen) were applied. Basis and aluminum saturation and $\mathrm{pH}$ were evaluated in 0-10 and 10-20 cm soil layer. In 0-10 cm soil layer, pH decreases 0.27 units per $100 \mathrm{~kg}$ ha $^{-1}$ year $^{-1}$ of nitrogen applied. In 10-20 cm soil layer, pH decreases of 5.3 to 4.7 and 4.6 with dose of nitrogen of 100 and $200 \mathrm{~kg} \mathrm{ha}^{-1}$ year $^{-1}$, respectively. Long-term nitrogen fertilization in native pasture with ryegrass introduction resulted in soil acidification until $20 \mathrm{~cm}$ soil depth.
\end{abstract}

KEYWORDS: acidity indicators, natural pasture, liming.

A pastagem nativa do bioma Pampa é a principal fonte nutricional utilizada na ovinocultura e pecuária de corte no estado do Rio Grande do Sul (SEBRAE/SENAR/FARSUL 2005). Por esse Estado possuir o primeiro $(22,9 \%)$ e o sexto (6,9\%) maior rebanho de ovinos e bovinos do Brasil (IBGE 2010), avanços no manejo dessa fonte de forragem são necessários para a sustentabilidade do setor. No entanto, as alterações antrópicas nos ecossistemas pastoris vêm descaracterizando esses ambientes, com diminuição de espécies desejáveis e da capacidade de suporte de carga animal do campo (SILVA et al. 2015).

A intensificação da produção em pastagens possibilita o atendimento da demanda de carne por aumentar a produtividade e reduzir a necessidade de exploração de novas áreas (CORSI et al. 2007). Para que tal processo ocorra, é necessário o uso de fertilizantes nitrogenados, uma vez que o nitrogênio (N) é um nutriente exigido em grande quantidade, indispensável para o desenvolvimento vegetativo (TARANET et al. 2017) e, consequentemente, aumento de produção da biomassa forrageira (SARMENTO et al. 2008). Como nesse bioma predominam espécies estivais (BOLDRINI 1997), faz-se necessária a inserção de espécies 
hibernais com alta produtividade, tais como o azevém (Lolium multiflorum Lam.). A fertilização nitrogenada e a introdução do azevém em campo nativo podem, então, ser uma alternativa para a exploração adequada do bioma Pampa.

Dentre as inúmeras fontes nitrogenadas utilizadas, a ureia ocupa destaque, por seu menor custo por unidade do nutriente entre os fertilizantes nitrogenados disponíveis (OTTO et al. 2017). A fertilização nitrogenada com adubos amoniacais e amídicos tem se mostrado, por sua vez, responsável pela aceleração do processo de acidificação do solo em pastagens (CAl et al. 2015), o qual é um processo contínuo em regiões onde a precipitação excede a evapotranspiração (BOLAN et al. 1991). A ureia possui potencial acidificante de $1 \mathrm{H}^{+}$por unidade de $\mathrm{N}$, enquanto que as fontes oriundas de sal de amônia possuem o dobro desse potencial (SUMNER 2009). A magnitude da acidificação do solo medida pelo decréscimo do pH depende principalmente da sua capacidade de tamponamento, sendo esta influenciada pela textura e pelo teor de matéria orgânica do solo (MOS) (BOLAN et al. 1991).

Não menos importante, no tocante ao equilíbrio de sistemas produtivos no longo prazo, o fator tempo deve ser contemplado (COSTA et al. 2010), visto que no Brasil, há uma cultura de degradação de pastagens ao longo do tempo (PERON \& EVANGELISTA 2004). Experimentos de longa duração que avaliam a dinâmica no sistema solo-planta em campo nativo no Brasil são escassos, sendo inexistentes no que se refere à fertilização nitrogenada.

Assim, o objetivo deste trabalho foi verificar como atributos químicos do solo relacionados com a acidificação de um Argissolo são afetados em longo prazo pelo pastejo contínuo em um campo nativo com introdução de azevém e fertilização nitrogenada em cobertura.

O experimento foi iniciado em 1996 na Estação Experimental Agronômica da Universidade Federal do Rio Grande do Sul, localizada no município de Eldorado do Sul $\left(30^{\circ} 05^{\prime} 52^{\prime \prime} \mathrm{S}, 51^{\circ} 39^{\prime} 08^{\prime \prime} \mathrm{O}\right.$ e altitude média de 46 metros), na região fisiográfica da Depressão Central do RS - Brasil. O clima é subtropical úmido "Cfa" de acordo com a classificação de Köppen, com temperatura, evapotranspiração de referência e precipitação média anual de $18,8^{\circ} \mathrm{C}, 1161$ e $1455 \mathrm{~mm}$, respectivamente (BERGAMASCHI et al. 2003). O solo é classificado como Argissolo Vermelho, com textura franco argilosa, possuindo $130 \mathrm{~g} \mathrm{~kg}^{-1}$ de argila na camada de $0-20 \mathrm{~cm}$.

O local foi caracterizado como uma sucessão secundária da pastagem natural. Em 1996, o solo foi fertilizado e sua acidez corrigida para pH 6,0. Naquele ano, o pastejo de bovinos de corte na pastagem natural foi iniciado com os tratamentos de doses anuais de nitrogênio em cobertura de 0,100 e $200 \mathrm{~kg} \mathrm{ha}^{-1}$ $a^{-1}$ de $\mathrm{N}$, na forma de ureia. $\mathrm{O}$ ajuste de carga de bovinos de corte foi efetuado para manter a oferta de forragem em $12 \%$. As doses de $\mathrm{N}$ foram aplicadas em duas vezes de 0,50 e $100 \mathrm{~kg} \mathrm{ha}^{-1}$, sendo a primeira aplicada em junho e a segunda em outubro de cada ano. De 2004 a 2006, o experimento foi submetido ao pastejo moderado de ovinos, sem aplicação de N. Em 2007, os tratamentos foram retomados, sendo a área pastejada por bovinos de corte. Nesse ano, a acidez do solo foi novamente corrigida para pH 6,0 e o azevém anual foi introduzido na área experimental em semeadura direta, sendo utilizados na base $300 \mathrm{~kg}$ ha $^{-1}$ de monoamônio fosfato (MAP) (12-52-00), totalizando 36 e $156 \mathrm{~kg} \mathrm{ha}^{-1}$ de $\mathrm{N} \mathrm{e} \mathrm{P}_{2} \mathrm{O}_{5}$, respectivamente, além das doses de $\mathrm{N}$ aplicadas em cobertura, correspondentes aos tratamentos. No inverno, a espécie predominante foi o azevém anual, enquanto, no verão, foram Paspalum notatum e Cynodon dactylon. Em 2010 as aplicações de $\mathrm{N}$ foram realizadas nos dias 30/06 e 02/11, havendo precipitação pluviométrica em torno de $15 \mathrm{~mm}$ nove e sete dias após as aplicações, respectivamente. Neste ano, a produção acumulada de matéria seca de forragem foi de $7.918,9.657$ e $14.144 \mathrm{~kg} \mathrm{ha}^{-1}$, enquanto a carga animal foi de 757, $895 \mathrm{e}$ $1.167 \mathrm{~kg} \mathrm{ha}^{-1}$ de peso vivo para as doses de $\mathrm{N}$ de 0,100 e $200 \mathrm{~kg} \mathrm{ha}^{-1}$ ano-1, respectivamente. $\mathrm{O}$ experimento foi conduzido em delineamento experimental de blocos ao acaso com duas repetições, em uma área de 3,11 hectares. As parcelas experimentais possuíam dimensão variando entre 0,3961 e 0,6587 ha.

Os atributos químicos na camada de $0-20 \mathrm{~cm}$ foram avaliados em uma amostragem realizada em agosto de 2010, sendo determinado a matéria orgânica do solo, fósforo e potássio extraíveis (Mehlich 1) e cálcio e magnésio trocáveis $\left(\mathrm{KCl} 1 \mathrm{~mol} \mathrm{~L}^{-1}\right)$ os valores encontrados foram: $18,2 \mathrm{~g} \mathrm{~kg}^{-1}, 18,6 \mathrm{mg} \mathrm{dm}^{-3}, 0,31$ $\mathrm{cmolc} \mathrm{kg}^{-1}, 1,45 \mathrm{cmolc}_{\mathrm{cg}} \mathrm{kg}^{-1}, 83 \mathrm{cmolc}_{\mathrm{kg}}{ }^{-1}$, respectivamente.

Os atributos químicos analisados relacionados com acidez do solo foram $\mathrm{pH}$ em água (1:1), saturação por bases e saturação por alumínio (TEDESCO et al. 1995). Estes atributos foram determinados em amostras compostas por dez subamostras das camadas de 0-10 e 10-20 cm. Uma área de campo nativo sem pastejo localizada a cerca de $500 \mathrm{~m}$ do experimento também foi amostrada. Essa área foi pastejada até 1980, quando o pastejo foi suspenso.

Os resultados obtidos foram submetidos à análise de variância (ANOVA) e, quando significativas $(p<0,10)$, as médias foram comparadas pelo teste Tukey $(p<0,10)$ pelo aplicativo computacional SISVAR 
(FERREIRA 2011).

A calagem resultou em maior valor de $\mathrm{pH}$ e saturação por bases e menor saturação por alumínio na camada de $0-10 \mathrm{~cm}$ de solo no tratamento sem aplicação de $\mathrm{N}$, em comparação com a área de referência (Tabela 1). No entanto, a aplicação de $\mathrm{N}$ acidificou o solo em ambas as camadas $(0-10$ e $10-20 \mathrm{~cm})$, medida pelo efeito inverso ao que aconteceu com a calagem. Na camada de $0-10 \mathrm{~cm}$, para cada $100 \mathrm{~kg} \mathrm{ha}^{-1} \mathrm{ano}^{-1}$ de $\mathrm{N}$ aplicado em cobertura, $\mathrm{o} \mathrm{pH}$ decresceu 0,27 unidades, diferindo entre os tratamentos $(\mathrm{p}<0,10)$. Da mesma forma, na camada de $10-20 \mathrm{~cm}$ o pH decresceu de 5,3 até 4,7 e 4,6 com a aplicação de $\mathrm{N}$ na dose moderada (100 kg ha-1 ano-1) e alta (200 kg ha-1 ano-1), respectivamente. A acidificação do solo por adição de $\mathrm{N}$ na camada de $0-20 \mathrm{~cm}$ foi observada por CAl et al. (2015), no cultivo de trigo e milho em um Cambissolo, com dose de $300 \mathrm{~kg} \mathrm{ha}^{-1} \mathrm{ano}^{-1}$ de $\mathrm{N}$ na forma de ureia.

Tabela 1. Atributos indicadores de acidez em Argissolo, com pastejo contínuo de pastagem natural e introdução de azevém, submetido a diferentes doses de nitrogênio em cobertura.

Table 1. Acidity indicators attributes in Ultisol, with continuous grazing of natural pasture and ryegrass introduction, under different nitrogen fertilizer doses.

\begin{tabular}{lclccc}
\hline \multirow{2}{*}{$\begin{array}{l}\text { Atributo de } \\
\text { acidez do solo }\end{array}$} & \multirow{2}{*}{$\begin{array}{c}\text { Camada de } \\
\text { solo }(\mathrm{cm})\end{array}$} & \multirow{2}{*}{$\begin{array}{l}\text { Área de } \\
\text { referência }\end{array}$} & \multicolumn{3}{c}{$\begin{array}{c}\text { Doses de nitrogênio } \\
\mathrm{kg} \mathrm{ha}^{-1} \mathrm{ano}^{-1}\end{array}$} \\
\cline { 4 - 6 } & & & 0 & 100 & 200 \\
\hline \multirow{2}{*}{$\mathrm{pH}$ em água } & $0-10$ & $5,3 \mathrm{aB}$ & $5,6 \mathrm{aA}$ & $5,4 \mathrm{aB}$ & $5,1 \mathrm{aC}$ \\
& $10-20$ & $5,1 \mathrm{bB}$ & $5,3 \mathrm{bA}$ & $4,7 \mathrm{bC}$ & $4,6 \mathrm{bC}$ \\
\multirow{2}{*}{ Saturação por Al ${ }^{3+}(\%)$} & $0-10$ & $12 \mathrm{bB}$ & $0 \mathrm{bD}$ & $8 \mathrm{bC}$ & $25 \mathrm{bA}$ \\
& $10-20$ & $31 \mathrm{aC}$ & $16 \mathrm{aD}$ & $35 \mathrm{aB}$ & $54 \mathrm{aA}$ \\
Saturação por bases (\%) & $0-10$ & $34 \mathrm{aC}$ & $53 \mathrm{aA}$ & $47 \mathrm{aB}$ & $26 \mathrm{aD}$ \\
& $10-20$ & $20 \mathrm{bB}$ & $34 \mathrm{bA}$ & $21 \mathrm{bB}$ & $12 \mathrm{bC}$ \\
\hline
\end{tabular}

Médias seguidas pela mesma letra maiúscula nas linhas e minúscula nas colunas não diferem significativamente pelo teste de Tukey $(p<0,10)$.

A maior acidez $(p<0,10)$, encontrada com as doses de $\mathrm{N}$ na camada de $0-10 \mathrm{~cm}$ (Tabela 1$)$, resulta do efeito acidificante da ureia, como visto anteriormente. Como consequência, a saturação por bases na camada de $0-10 \mathrm{~cm}$ variou de 26 a 53\%, e na camada de $10-20 \mathrm{~cm}$ variou de 12 a $34 \%$. Na maior dose de $\mathrm{N}$, a saturação por bases foi menor do que na área referência. Na saturação por alumínio houve efeito inverso, sendo maior com o aumento da dose de $\mathrm{N}$. Do ponto de vista de nutrição de plantas, a saturação por alumínio é mais prejudicial às plantas do que o efeito individual do $\mathrm{pH}$ (KOYAMA et al. 2001).

A fonte nitrogenada acidifica de forma diferenciada o solo ao longo do tempo, conforme reportado por NOBLE et al. (2008), onde $\mathrm{N}^{-\mathrm{NO}_{3}-}$ resultou em acidificação de $5,6 \mathrm{kmol} \mathrm{H}^{+}$ha-1 ano-1 um impacto menor quando comparado com N-NH $4^{+}$, que alcançou valores de $16,6 \mathrm{kmol} \mathrm{H}^{+} \mathrm{ha}^{-1}$ ano-1. No entanto, esses efeitos ocorreram com pastejo simulado em uma região onde a evapotranspiração excede em quase duas vezes a precipitação.

A lixiviação de nitrato só causa decréscimo permanente de $\mathrm{pH}$ se o nitrogênio adicionado ao solo for de fonte amídica ou amoniacal. As perdas por lixiviação desse ânion pode ser de 60 a $90 \mathrm{~kg} \mathrm{ha}^{-1} \mathrm{ano}^{-1}$ de N (BOLAN et al. 1991). Esse efeito ocorre com maior intensidade em solos com baixa capacidade de tamponamento, pois o processo de acidificação depende dos teores de argila e matéria orgânica (CHIEN et al. 2010). Assim, após 20 anos aplicando $37 \mathrm{~kg} \mathrm{ha}^{-1} \mathrm{ano}^{-1}$ de $\mathrm{N}$, na forma de ureia em um solo arenoso, BERG (1986) verificou um decréscimo de $\mathrm{pH}$ do solo de 6,7 para 5,3 na camada de $0-5 \mathrm{~cm}$, concluindo com a necessidade de aplicar $1,8 \mathrm{~kg} \mathrm{CaCO} 3$ para corrigir a acidez gerada por $\mathrm{kg}$ de $\mathrm{N}$ aplicado.

É importante frisar a participação da umidade do solo nos processos de hidrólise e nitrificação do Nureico (SUN et al. 2008). Em casos de baixa umidade, na ocasião da deposição da ureia no solo, pode haver perdas consideráveis de $\mathrm{N}$ devido à volatilização de amônia $\left(\mathrm{NH}_{3}\right)$, visto que a primeira fase da

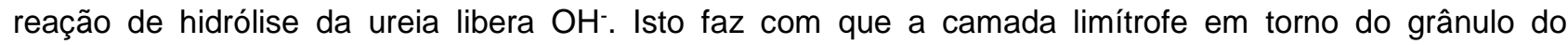
fertilizante se alcalinize, volatilizando a amônia gerada em meio básico. A quantidade de $\mathrm{N}$ perdida por volatilização após a aplicação de ureia sobre a superfície do solo pode atingir valores extremos, próximos a $80 \%$ do N aplicado (BERNARDI et al. 2010), sendo mais comum valores entre 16 e $40 \%$ (WERNECK et al. 2012, SOARES FILHO et al. 2015). A eficiência da ureia é aumentada se ocorrer chuva de $5 \mathrm{~mm}$ ou mais, até dois dias após a sua aplicação (WHITEHEAD 1995). FONTOURA \& BAYER (2010) observaram perda de $22,7 \%$ com a ureia permanecendo até 10 dias na superfície do solo antes que ocorresse uma chuva. 
Entretanto, essa perda foi diminuída para 2,1\% quando a chuva ocorreu até o segundo dia após a aplicação do fertilizante. No presente experimento, por não haver irrigação complementar, é recorrente a aplicação de ureia em condições de falta de umidade do solo por longos períodos. No ano de avaliação deste trabalho (2010), as chuvas ocorreram nove e sete dias após a primeira e segunda aplicação, respectivamente, com prováveis perdas de $\mathrm{N}$ na forma de $\mathrm{NH}_{3}$.

A maior produção de massa de forragem com as doses de $N$ (CARASSAl et al. 2008) e, consequentemente, a maior quantidade de animais em pastejo aumentam a mineralização de resíduos de plantas e de dejetos animais, acidificando o solo (SARMENTO et al. 2008), decorrente da liberação de ácidos orgânicos com a decomposição da liteira (RAMZANI et al. 2016). Assim, o entendimento do processo de retroalimentação é indispensável em um sistema de produção que busca atingir o equilíbrio em longo prazo.

A adubação nitrogenada de longo prazo com ureia em solos de campo nativo com introdução de azevém acidificou o solo até a profundidade de $20 \mathrm{~cm}$. No entanto, a dose moderada de $100 \mathrm{~kg} \mathrm{~N} \mathrm{ha}^{-1} \mathrm{ano}^{-1}$ permite que haja utilização mais intensiva da pastagem, mantendo alguns indicadores de acidez semelhantes à área de referência.

\section{REFERÊNCIAS}

BERG WA. 1986. Effect of 20 years of low N rate pasture fertilization on soil acidity. Journal of Range Management 39 : $122-124$.

BERGAMASCHI H et al. 2003. Clima da Estação Experimental da UFRGS (e Região de Abrangência). Porto Alegre: UFRGS. 77p.

BERNARDI ACC et al. 2010. Volatilização de amônia, produção de matéria seca e teores foliares de $\mathrm{N}$ do azevém adubado com fontes nitrogenadas. São Carlos: Embrapa. 8p.

BOLAN NS et al. 1991. Processes of soil acidification during nitrogen cycling with emphasis on legume based pastures. Plant and Soil 134: 53-63.

BOLDRINI II. 1997. Campos do Rio Grande do Sul: caracterização fisionômica e problemática ocupacional. Porto Alegre: UFRGS. 39p.

CAI Z et al. 2015. Intensified soil acidification from chemical $\mathrm{N}$ fertilization and prevention by manure in an 18-year field experiment in the red soil of southern China. Journal of Soils and Sediments 15: 260-270.

CARASSAI IJ et al. 2008. Recria de cordeiras em pastagem nativa melhorada submetida à fertilização nitrogenada. I. Dinâmica da pastagem. Revista Brasileira de Zootecnia 37: 1338-1346.

CHIEN SH et al. 2010. Liming requirement for nitrogen fertilizer-induced soil acidity: a new examination of AOAC guidelines. Better Crops with Plant Food 94: 8-9.

CORSI M et al. 2007. Nitrogênio e enxofre em pastagens. In: YAMADA T et al. Nitrogênio e enxofre na agricultura brasileira. Piracicaba: IPNI Brasil. p. 487-518.

COSTA SEVGA et al. 2010. Patterns in phosphorus and corn root distribution and yield in long-term tillage systems with fertilizer application. Soil \& Tillage Research 109: 41-49.

FERREIRA DF. 2011. Sisvar: a computer statistical analysis system. Ciência e Agrotecnologia 35: 1039-1042.

FONTOURA SMV \& BAYER C. 2010. Ammonia volatilization in no-till system in the south-central region of the state of Paraná, Brazil. Revista Brasileira de Ciência do Solo 34: 1677-1684.

IBGE. 2010. Instituto Brasileiro de Geografia e Estatística. Produção da Pecuária Municipal. Disponível em: http://www.ibge.gov.br/home/estatistica/economia/ppm/2010/default_pdf.shtm. Acesso em: 23 ago. 2017.

KOYAMA H et al. 2001. Brief exposure to low-pH stress causes irreversible damage to the growing root in Arabidopsis thaliana: pectin-Ca interaction may play an important role in proton rhizotoxicity. Journal of Experimental Botany 52 : 361-368.

NOBLE AD et al. 2008. Soil acidification and carbon storage in fertilized pastures of Northeast Thailand. Geoderma 144: 248-255.

OTTO R et al. 2017. Ammonia volatilization from nitrogen fertilizers applied to sugarcane straw. Revista Ciência Agronômica 48: 413-418.

PERON AJ \& EVANGELISTA AR. 2004. Degradação de pastagens em regiões de cerrado. Ciência e Agrotecnologia 28: 655-661.

RAMZANI PMA et al. 2016. Iron biofortification of wheat grains through integrated use of organic and chemical fertilizers in pH affected calcareous soil. Plant Physiology and Biochemistry 104: 284-293.

SARMENTO P et al. 2008. Atributos químicos e físicos de um argissolo cultivado com Panicum maxicum Jacq. cv. IPR36 Milênio, sob lotação rotacionada e adubado com nitrogênio. Revista Brasileira de Ciência do Solo 32: 183-193.

SEBRAE/SENAR/FARSUL. 2005. Diagnóstico de sistemas de produção de bovinocultura de corte no estado do Rio Grande do Sul. Porto Alegre: SENAR. 144p.

SILVA SC et al. 2015. Ecophysiology of C4 forage grasses-understanding plant growth for optimising their use and management. Agriculture 5: 598-625.

SOARES FILHO CV et al. 2015. Ammonia volatilization losses in Tanzania grass fertilized with urea. Revista Brasileira de Saúde e Produção Animal 16: 253-264. 
SUMNER ME. 2009. Soil acidification. In: VERHEYE WH. Land use, land cover and soil sciences. Brisbane: EOLSS. v. 7. $235 \mathrm{p}$.

SUN X et al. 2008. Fertilizer nitrogen and factors affecting pasture responses. The Open Agriculture Journal 2: 35-42.

TARANET P et al. 2017. Growth and yield response of glasshouse- and field-grown sweetpotato to nitrogen supply. Nutrient Cycling in Agroecosystem 108: 309-321.

TEDESCO JM et al. 1995. Análise de solo, plantas e outros materiais. 2.ed. Porto Alegre: UFRGS. 174p. (Boletim técnico 5).

WERNECK CG et al. 2012. Volatilização de amônia proveniente de ureia com zeólita natural. Pesquisa Agropecuária Brasileira 47: 466-470.

WHITEHEAD DC. 1995. Volatilization of ammonia. In: WHITEHEAD DC. Grassland nitrogen. Wallingford: CAB International. p. 152-179. 